\title{
Solar-cell based powering of a node for traffic monitoring
}

\author{
Ondrej Karpis ${ }^{1}$ \\ ${ }^{1}$ (Department of Technical Cybernetics, Univerzity of Zilina, Slovakia)
}

\begin{abstract}
This paper discusses the possibility of using solar cell for supplying energy to a node designated to monitor the traffic. Analysis of the energy requirements of each part of the node is crucial for proper design of the power supply. As energy storage is used an ultracapacitor. The paper contains measurements of energy provided by solar cell under realistic conditions. The results indicate the possibility of using a solar cell to power the node for most of the year.
\end{abstract}

Keywords: power supply, solar cell, traffic monitoring, ultracapacitor, wireless sensor network

\section{INTRODUCTION}

Wireless sensor networks (WSN) technologies are among the most popular today. Such a network consists of a large number of relatively simple elements, which are located in an area of interest and communicate with each other through wireless technology. Area covered by a wireless sensor network can range from a few square meters to square kilometers. Each network element (node) is able to obtain the required data, process them, and communicate with other network elements. The rapid development of microelectronics, especially in the field of micro-electro-mechanical sensors (MEMS), allows the use of wireless networks in an increasingly broad range of applications.

Main areas of WSN application are:

- Industry: monitoring of technological processes, security systems and more recently production management [1],

- Military applications: monitoring the number and movement of troops and military equipment, protection of important areas, sniper detection, etc. [2],

- Health: monitoring of high-risk groups of patients, the automatic administration of drugs to patients based on their current health status, monitoring the movement of medical personnel and supplies, etc.,

- Environmental monitoring: improvement in weather forecasting, monitoring of emissions, protection of nature from unauthorized activities and so on [3],

- Transport: adaptive control of intersections, parking systems, congestion detection and real-time navigation of drivers to unblocked roads, vehicle classification, etc. [4].

A large share of the WSN market have devices for measuring and recording energy and water consumption in households. These applications are usually known as "Smart Grid." The Department of Technical Cybernetics at the University of Zilina has for many years engaged in the issue of using wireless networks in the field of intelligent transport systems. For proper operation of these systems is necessary to know the traffic situation in real time. Traditional means of obtaining the necessary information include traffic sensors built right into the road: inductive loops, piezo-electric cables, pneumatic tubes and others. These systems are very reliable in detecting passing vehicles $(>97 \%)$. In order to make use of intelligent transport systems to the maximum extent it is necessary to install the sensors on all major roads as well as on many local roads. But installation and maintenance of these traditional systems is very costly and causes serious traffic disturbance, resulting in a further increase in price. For these reasons such sensors are not suitable for use on a larger scale. Into the second group of sensors belong sensors that do not require incorporation directly into the road. Installation or maintenance of such systems will not cause traffic disruption. This includes radar systems, video systems, infrared and ultrasonic sensors as well as passive acoustic arrays. The major disadvantage of video and infrared sensor systems is their dependence on the weather. Radar and passive acoustic systems have problems with standing or slow-moving vehicles. Advances in technology allowed the development of new types of sensors - miniature magnetometers, which are capable of measuring the intensity of the magnetic field. Magnetically conductive material (metal in vehicles) affects the intensity of the geomagnetic field in its vicinity. These changes can be detected by magnetometers at a relatively large distance from the vehicle. Magnetometers can be successfully used to measure all the parameters necessary to monitor the traffic: the number of vehicles, their speed, classification, occupancy of parking spaces. Their other advantages include low cost, small size, low power consumption and high reliability. Magnetometers must be installed in the road (or sidewalk), but this action is of incomparably smaller scale than in the case of induction loops. Ability of magnetometers to detect 
vehicles is comparable with induction loops. For these reasons, most traffic monitoring systems are based on magnetometers nowadays. Successful use of WSN for traffic monitoring is dependent on resolving other issues: power supply for the node and communication between the nodes. Both problems are closely related. Largest consumer of energy is the communication subsystem of the node. Ideally, communication should be fast and long distance. It can be achieved in practice, but only at the cost of high energy consumption. Therefore, the most widely used communication modules operate within a range from 30 to $300 \mathrm{~m}$ with transmit power of 0 to $10 \mathrm{dBm}$ and receiver sensitivity of -90 to $-102 \mathrm{dBm}$. The transceivers usually operate in the ISM bands 443 $\mathrm{MHz}, 886 / 916 \mathrm{MHz}$ and $2.4 \mathrm{GHz}$. Transfer rate in most WSN applications is limited to 10-250 kbps. Reducing the consumption of the communication subsystem is possible by reducing data rates and transmission power and by smart controlling of the network: the network is active only at well-defined intervals and for a limited time.

\section{POWER SUBSYSTEM OF THE NODE}

In most WSN applications, the designers are faced with one fundamental problem - how to ensure network operation without operator intervention. Unattended operation of the sensor networks is required especially in applications deployed over a large area. Lifetime of the node can be seen as an operation time without the need for operator intervention. Node lifetime can be extended by increasing the capacity of the energy sources or by reducing the device consumption. Another option is to power the sensor system using some kind of energy harvesting. Today we meet with microcontrollers, whose consumption in active mode is below $100 \mathrm{uA} / \mathrm{MHz} @ 3 \mathrm{~V}$. The single battery with a capacity of $1000 \mathrm{mAh}$ can power a processing unit for up to 1 year. In real applications, the microcontroller is often in a sleep mode with a hundred times less power consumption. Then the limiting factor becomes the consumption of the communication module and battery selfdischarging. Primary cells and rechargeable batteries has recently faced with a significant improvement in parameters, especially the increase in energy density and reducing of self-discharging current. Power cells based on Li-SOCl2 (lithium thionyl chloride) have now an energy density of $1220 \mathrm{Wh} / 1$ and their self-discharge is less than $1 \%$ of capacity per year. Such cells are intended for equipment with a lifetime of about 20 years.

The second option, which is more and more to the forefront of interest, are systems for gathering energy from the environment in collaboration with rechargeable batteries or ultracapacitors. In the case of traffic monitoring, where sensors are embedded in the road, the most suitable energy source seems to be solar energy in conjunction with ultracapacitors. Recently, power system based on two ultracapacitors was developed at our department [5]. Charging of the main ultracapacitor can be using various sources of renewable energy (light, heat, vibration). This power system is ideal in those cases where it is not possible to use conventional energy sources, such as the monitoring of the environment [6].

\section{POWER REQUIREMENTS OF THE NODE}

Node prototype developed in our department is based on a 32-bit microcontroller STM32F100RB. The microcontroller has a core ARM Cortex-M3. Sensing part of the node consists from the sensor LSM303 containing 3-axis magnetometer and 3-axis accelerometer. The communication subsystem is based on the module MRF24J40MA (Microchip). The module operates in the ISM $2.4 \mathrm{GHz}$ band with data rates up to 256 $\mathrm{kb} / \mathrm{s}$ (normal mode) or $625 \mathrm{~kb} / \mathrm{s}$ (turbo mode) and communication range up to $120 \mathrm{~m}$. Large-capacity data storage is provided by a micro SD card. Motherboard dimensions are 49 × $33 \mathrm{~mm}$ (Fig. 1).
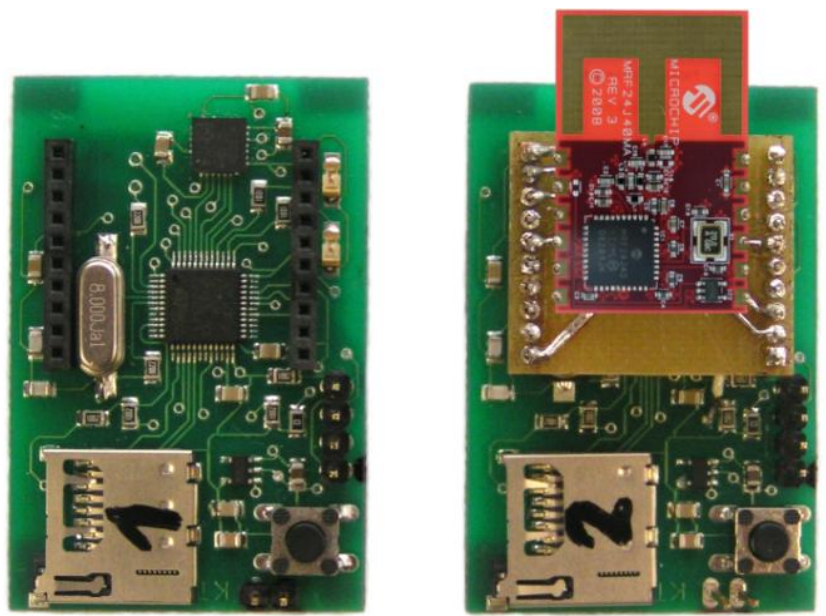

Fig.1 Prototype of the node for traffic monitoring 
Consumption of the entire system depends on several factors: the supply voltage level, the number of passing vehicles, method of processing and transmission of the measured data and the like. For this analysis we assume that the supply voltage is $3 \mathrm{~V}$. Consumption of the individual parts of the node is given in Table 1 .

Table 1 Consumption of node parts

\begin{tabular}{|l|l|l|}
\hline \multirow{2}{*}{ Microcontroller STM32F100RB } & Run mode@ $125 \mathrm{kHz}$ & $1.35 \mathrm{~mW}(0.45 \mathrm{~mA})$ \\
\cline { 2 - 3 } & Run mode @ 24 MHz & $36.6 \mathrm{~mW}(12.2 \mathrm{~mA})$ \\
\hline \multirow{2}{*}{ Magnetometer LSM303 } & Normal mode & $1.08 \mathrm{~mW}(0.36 \mathrm{~mA})$ \\
\cline { 2 - 3 } & Power down mode & $6 \mathrm{uW}(2 \mathrm{uA})$ \\
\hline \multirow{3}{*}{ Transceiver MRF24J40MA } & Transmit mode & $69 \mathrm{~mW}(23 \mathrm{~mA})$ \\
\cline { 2 - 3 } & Receive mode & $57 \mathrm{~mW}(19 \mathrm{~mA})$ \\
\cline { 2 - 3 } & Sleep & $6 \mathrm{uW}(2 \mathrm{uA})$ \\
\hline
\end{tabular}

Unfortunately, the magnetometer is not able to generate interrupt signal in the case of detection of the magnetic field change. Therefore the output of magnetometer must be continuously checked by the microcontroller (we cannot use sleep or stop mode). In this case the consumption of the microcontroller can be reduced only by lowering the main clock frequency. The microcontroller will use low frequency clock during monitoring of the magnetometer output and will increase the main clock after detection of the vehicle. Processing of the measured data will be done at maximum clock frequency, as it has the best ratio of energy consumption per instruction. Processed data (if any) will be transmitted through the wireless module every $10 \mathrm{~s}$. We assume a small amount of data to be transferred (up to $10 \mathrm{~B}$ ), which will contain information only on the number and type of vehicles detected. Time needed to transmit a packet consisting of 10 bytes of data and 11 bytes of packet header is $0.672 \mathrm{~ms} @ 250 \mathrm{kbps}$ (in the next calculation we will use $1 \mathrm{~ms}$ ). Let the time required to process the measured data be a $100 \mathrm{~ms}$. The microcontroller will be in a sleep mode for $9900 \mathrm{~ms}$ and $100 \mathrm{~ms}$ in the normal mode during the 10-second interval. Magnetometer will be all the time in the normal mode. Transceiver is $9999 \mathrm{~ms}$ in the sleep mode and only $1 \mathrm{~ms}$ in the transmit mode. The average consumption of the device is $2.795 \mathrm{~mW}$. For one hour the node consumes about $10 \mathrm{~J}$ of energy. This calculation does not include efficiency of the power supply unit, so the final consumption will be somewhat larger.

\section{SOLAR CELL AS AN ENERGY SOURCE}

We plan to use the solar cell SMH-8-0450 to power the node. Its active area is about $23 \mathrm{~cm}^{2}$. For energy storage will be used ultracapacitor of capacity 40F/5.6V. Assuming that the ultracapacitor voltage is between 2 to $5 \mathrm{~V}$, the ultracapacitor can store about $180 \mathrm{~J}$ of energy. This amount of energy must be sufficient to operate the node during the period when it is not possible to exploit solar energy. If the estimated hourly consumption of the node is $10 \mathrm{~J}$, fully charged ultracapacitor should be able to power the node for 18 hours. This should be sufficient to overcome periods without sunlight even in the winter months.

The time required to charge the capacitor is as follows:

$t=\frac{1000 . E}{P_{M P P}}$

where $t$ is time $[\mathrm{s}], \mathrm{E}$ is the energy $[\mathrm{J}]$ and $\mathrm{P}_{\mathrm{MPP}}$ is the maximum power of the solar cell under ideal conditions $[\mathrm{mW}]$.

According to the datasheet, the maximum power of the solar cell is $418 \mathrm{~mW} \pm 10 \%$. The time required to fully charge the ultracapacitor will then be about 7 minutes and 10 seconds. It should be noted that these results apply only when conditions are ideal (i.e. when the power density of sunlight is $1 \mathrm{~kW} / \mathrm{m} 2$ ). In Slovakia, such conditions occur only about two months of a year (June and July). We assume that extension of the ultracapacitor charging time due to weaker solar radiation should not cause any serious problem.Several measurements were made to verify the ability of the selected solar panel to recharge the ultracapacitor. The solar cell was placed in a horizontal position at a place that is not shielded by any object. Measurements were carried out on 14 to 18 March 2013 in town Prievidza (GPS coordinates: N 4846'22.11", E $\left.18^{\circ} 37^{\prime} 01.76^{\prime \prime}\right)$. The measurements were carried out during different weather: cloudy and sunny. Solar cell was loaded with a constant load of $2.2 \mathrm{kOhm}$. The voltage generated by the cell was sampled once per second. Of course, constant load is not suitable to maximize the energy produced by the solar cell. To maximize its performance it would be necessary to use maximum power point tracking. However, it is possible to estimate the maximum energy generated by the cell using a solar cell model. Solar cells can be modeled in different ways. Perhaps the most commonly used is single diode model (SDM) [7]. The use of models is limited by the fact that manufacturers of solar panels do not provide the parameters necessary for these models. There are different approaches to derive the V-I characteristics of a solar cell only by the manufacturer provided parameters [8]. One option is to use an ideal single diode model (ISDM). This model neglects the influence of 
series and shunt resistances in the classic SDM. For our purposes, the accuracy of this model should be sufficient.

The equation to calculate the solar cell current is as follows:

$I=I_{S C}\left(1-\exp \left[\frac{\ln \left(1-I_{M P P} / I_{S C}\right) \cdot\left(V-V_{O C}\right)}{V_{M P P}-V_{O C}}\right]\right)$

where $\mathrm{I}_{\mathrm{SC}}$ is the short circuit current, $\mathrm{V}_{\mathrm{OC}}$ is open circuit voltage, $\mathrm{I}_{\mathrm{MPP}}$ and $\mathrm{V}_{\mathrm{MPP}}$ are current and voltage at maximum power point respectively and $\mathrm{V}$ is the actual voltage generated by the cell.

The formula can be used to obtain V-I characteristics of the solar cell under ideal conditions (intensity of radiation $1 \mathrm{~kW} / \mathrm{m}^{2}$, temperature $25^{\circ} \mathrm{C}$ ). Characteristics at different intensity of sunlight are obtained by moving the original characteristic along the axis I. Influence of temperature on the data shape is neglected. Using the voltage measured between the solar cell outputs we can calculate the current flowing through the constant load. The difference between the measured current and the current calculated using (2) represents a shift of the V-I characteristics due to the different intensity of solar radiation. In the shifted characteristic we can found the point corresponding to maximum power.

Figure 2 shows the maximum available power during two days with different weather.

Table 2 shows the total energy produced by the cell per day and the average time required to recharge the ultracapacitors. Sunrise on both days was at about 6:00 AM and sunset at 5:50 PM (i.e. $11 \mathrm{~h}$. $50 \mathrm{~min}$ of sunlight). The values obtained from the cell placed inside of the building about $30 \mathrm{~cm}$ from the large east facing window are provided for comparison purposes.
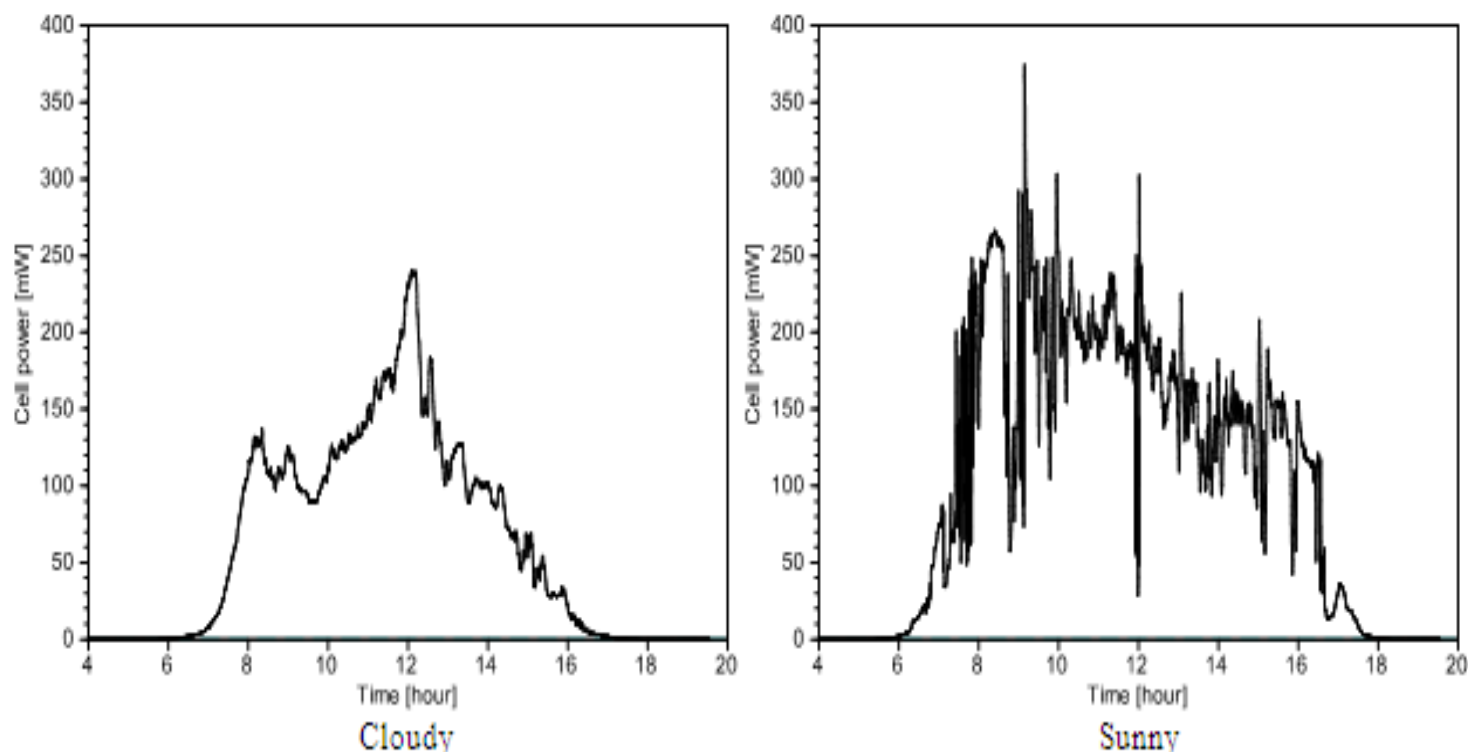

Fig.2 Available power from the solar cell

Table 2 Performance of the solar cell

\begin{tabular}{|l|c|c|c|c|}
\hline & \multicolumn{2}{|c|}{ Outside of building } & \multicolumn{2}{c|}{ Inside of building } \\
\hline Weather & Total energy & Recharge time & Total energy & Recharge time \\
\hline Cloudy & $3475 \mathrm{~J}$ & $37 \mathrm{~min}$. & $182 \mathrm{~J}$ & 1 day \\
\hline Sunny & $5851 \mathrm{~J}$ & $22 \mathrm{~min}$. & $269 \mathrm{~J}$ & $7 \mathrm{~h} .56 \mathrm{~min}$. \\
\hline
\end{tabular}

It is clear that the solar cell inside the building is of limited use. The situation outside of the building is much better, but it should be noted that the calculated time required to recharge the ultracapacitor would actually be greater, since the calculation does not include the power supply efficiency and also consumption of the node itself. Based on the measurements we assume that the solar cell will be able to charge the ultracapacitor to the maximum even in winter months. Thus, the system should be able to drive the node throughout the year. An open question is the impact of the snow, mud and dust on the solar cell efficiency. We do not assume that the solar cell will be completely covered with mud in the summer. In the winter it is more probable that the solar cell will be covered with a layer of snow or ice. In this case the cell will probably not be able to generate enough energy for continuous operation of the node and we will have to consider other options for its powering. 


\section{CONCLUSION}

The power subsystem is one of the most important parts of the node in wireless sensor networks. The functionality of the node depends on an adequate supply of energy. Node lifetime is often limited by the lifetime of its energy source. The use of non-renewable energy sources can significantly reduce the lifetime of the device. It is therefore important to focus on renewable energy sources such as solar energy. Analysis of energy requirements of the node for traffic monitoring together with the measurements of energy provided by the solar cell indicate the possibility of a continuous powering of the node using solely solar energy. Ultracapacitor is used to bridge periods when solar energy is not available. The possibility of using such a system in the winter months is yet to be verified. If the solar cell cannot provide enough energy, we plan to use a thermoelectric generator as a supplementary energy source.

\section{REFERENCES}

[1]. A. Herms, E. Nett, S. Schemmer, Real-time mesh networks for industrial applications, Proceedings of 17th International Federation of Automatic Control World Congress (IFAC'08), Seoul, Korea, 2008, 9314-9319.

[2]. M. Maroty et al., Shooter Localization in Urban Terrain, Computer, v.37, n.4, 2004, 60-61.

[3]. J. Papan, M. Jurecka, J. Puchyova, WSN for forest monitoring to prevent illegal logging, Proceedings of FedCSIS 2012, Wroclaw, Poland, 2012, 809-812.

[4]. O. Karpis, Sensor for Vehicles Classification, Proceedings of FedCSIS 2012, Wroclaw, Poland, 2012, 785-789.

[5]. M. Kochlan, P. Sevcik, Supercapacitor power unit for an event-driven wireless sensor node, Proceedings of FedCSIS 2012, Wroclaw, Poland, 2012, 791-796.

[6]. J. Micek, J. Kapitulik, WSN sensor Node for Protected Area Monitoring, Proceedings of FedCSIS 2012, Wroclaw, Poland, 2012, 831-835.

[7]. J. Nelson, The Physics of Solar Cells (Imperial College Press, 2003).

[8]. E. Matagne, R. Chenni, R. El Bachtiri, A photovoltaic cell model based on nominal data only, Proc. IEEE International Conference on Power Engineering, Energy and Electrical Drives, 2007, 562-565. 